\title{
Demixing and tetratic ordering in some binary mixtures of hard superellipses
}

\author{
Sakine Mizani ${ }^{1}$, Péter Gurin ${ }^{2}$, Roohollah Aliabadi ${ }^{3}$, Hamdollah Salehi $^{1}$ and \\ Szabolcs Varga ${ }^{2 *}$
}

${ }^{1}$ Department of Physics, Faculty of Science, Shahid Chamran University of Ahvaz, Ahvaz, Iran

${ }^{2}$ Institute of Physics and Mechatronics, University of Pannonia, P.O. Box 158, Veszprém, H-8201, Hungary

${ }^{3}$ Department of Physics, Faculty of Science, Fasa University, 74617-81189

Fasa, Iran

\begin{abstract}
We examine the fluid phase behaviour of binary mixtures of hard superellipses using the scaled particle theory. The superellipse is a general two-dimensional convex object, which can be tuned between elliptical and rectangular shapes continuously at a given aspect ratio. We find that the shape of the particle affects strongly the stability of isotropic, nematic and tetratic phases in the mixture even if the side-lengths of both species are fixed. While the isotropicisotropic demixing transition can be ruled out using the scaled particle theory, the first order isotropic-nematic and the nematic-nematic demixing transition can be stabilized with strong fractionation between the components. It is observed that the demixing tendency is strongest in small rectangle-large ellipse mixtures. Interestingly, it is possible to stabilize the tetratic order at lower densities in the mixture of hard squares and rectangles where the long rectangles form nematic phase, while the squares stay in tetratic order.
\end{abstract}

*Corresponding author: vargasz@almos.uni-pannon.hu 


\section{Introduction}

With the rapid development of new colloidal and granular materials, the tailoring technique of the nano- and microparticles has created new shapes of particles (e.g. superballs, lenses, stars) which exhibit very interesting percolation, glass formation, jamming behaviour and phase transitions [1-3]. To understand the observed structures and phase behaviours, several geometrical properties (curvature, volume, contact distance, excluded volume,...) have to be determined since many macroscopic properties depends on the details of the shape and the size of the particles [4].

One of the phase transitions of rod-like particles, where the shape really matters, is the nematic-smectic A phase transition. In the system of hard particles, which consists of cylinders or spherocylinders, it is observed that the smectic A phase is stable if the particles are at least moderately elongated [5-6], while the smectic A phase is totally absent in the system of hard ellipsoids [7]. To understand this strange behaviour it was useful to introduce new geometrical models for the particles, which interpolate between the cylindrical and ellipsoidal shapes. In this regard the superellipsoidal [8-9] and the sphero-ellipsoidal [10] shapes have served new information about the importance of the roundness of the central part and the end parts of the particles, respectively. The other example is the suspension of colloidal superballs, where the shape is between spherical and cubic, exhibits richer phase behaviour than those of spheres or cubes. In the suspension of superballs, new solid phases are observed including a solid-solid transition between a plastic and a rhombohedral crystal [11]. The monolayer of colloidal superballs behaves very similarly because a phase transition between a hexagonal rotator crystal phase and a rhombic crystal phase emerges [12]. To explain this solid-solid phase transition it is necessary to go beyond the two-dimensional (2D) hard-square model, because hard squares form only tetratic and square crystal phases [13-15]. Avendaño and Escobedo [16] have performed Monte Carlo simulations with a rounded hard-square model, which interpolates between squares and disks, to examine the effect of roundness on the stability of crystal phases. They showed that the roundness of the vertices is crucial in the formation of rotor crystal phase [16]. With changing the shape of the 2D particles the simulation studies and the vibration experiments revealed the existence of new phases such as the triatic phase of triangles, the tetratic phase of squares and the hexatic phase of hexagons, which are intermediate between fluid and solid phases [17-22]. Interestingly the system of hard pentagons does not form intermediate phases between the fluid and solid phases [23]. The common feature 
of these intermediate and ordered phases is the lack of true long-range order, which is manifested in quasi-long-range bond orientational and translational orders. Therefore, the transition from a fluid to an intermediate phase is governed by defect-mediated KosterlitzThouless mechanism [24]. Depending on the shape of hard particles, the order of 2D phase transitions can be either first order or continuous. For example, both the fluid-tetratic and the tetratic-solid phase transitions are continuous in the system of hard squares [23], while the hard disks undergo a first-order fluid-hexatic and a continuous hexatic-solid phase transitions with compression [25].

The phase behaviour of 2D hard body systems can be enriched by the stretching of spherical particles into one direction, which changes the particles to have ellipsoidal shape [26]. Confining them into a very narrow slit-like pore, it is possible to examine the ordering properties of a monolayer, which corresponds to the quasi-two-dimensional fluid of hard ellipses [27, 28]. Carbon nanotubes, viruses and DNA particles can be also confined into a narrow slit or can be absorbed on a surface, where they form a monolayer [29-31]. These systems can mimic the phase behaviour of 2D hard rectangles. A common feature of the ellipse and the rectangle systems is that a quasi-long-range orientiational order (nematic phase) emerges with increasing density if the particles are sufficiently elongated [32, 33]. The nature of the phase transition from the isotropic fluid phase to a nematic phase was shown to be continuous via a Kosterlitz-Thouless disclination unbinding type mechanism in the system of hard needles [34]. However, later studies revealed that the isotropic-nematic phase transition can be even first order $[32,35]$. Therefore the order of the transitions strongly depends on the details of the particle-particle interactions [35-39]. The important difference in the nematic ordering of hard ellipses and rectangles is that the minimal shape anisotropy (the ratio of the lengths of major and minor axes) required to stabilize the nematic phase is only 2.4 for ellipse [40], while it must be more than 7 for rectangular shapes [33,41]. Interestingly the vibration experiment with granular hard rectangles detected almost the same threshold for the stabilization of the nematic ordering, namely there is no $2 \mathrm{D}$ nematic for shape anisotropies lower than 7.3 [42], but the isotropic fluid phase changes continuously to the tetratic phase. This indicates that the tetratic ordering can preempt the nematic one in the system of rectangles, while the tetratic ordering is completely missing in hard ellipse systems.

To understand the differences occurring in the phase behaviours of hard disks and hard squares and in those of hard ellipses and hard rectangles it is necessary to define a hard body model which can be tuned between the two limiting shapes. In this regard, the hard superellipse 
model is a good candidate as it interpolates between ellipse and rectangle shapes. If the lengths of both sides of the superellipse are equal, we get the 2D superball model, which interpolates between a disk and a square. Along this line the first milestone was the exact determination of the maximal packing arrangement and the corresponding maximal packing fraction of hard superdisks [43]. Later, the jamming properties [44] and the kinetics of randomly packed superdisks [45] were also examined. These studies were extended to the binary mixtures of hard superdisks to locate the jammed state [46]. Regarding the hard superellipses we are only aware of the contact point calculations [47] and the percolation threshold study of the overlapping superellipses [48]. To study the phase behaviour of hard superellipses, a fast and accurate overlap check between two superellipses has to be devised to perform the simulations, while the excluded distances and areas are the key quantities in the mean-field theories such as the Onsager-theory and the scaled particle theory. The determination of these quantities is not trivial even for the hard ellipses $[49,50]$. The issues of the excluded area calculation for convex and concave $2 \mathrm{D}$ objects are considered in two recent publications [51, 52]. In our present study we show that the exclude area between two different superellipses having different sizes and shapes can be determined analytically.

It is a well-known fact that the phase behaviour of $2 \mathrm{D}$ mixtures is richer than that of one-component systems if the size and the shape of the components differ substantially [53]. It is found that very small amount of small disks can destabilize the hexatic phase of large disks and the transition becomes first order between the fluid and solid phases [54]. The destabilizations of the crystalline and the tetratic phases are predicted in size-polydisperse hard disk and in length-polydisperse hard rectangle systems [55, 56]. Strong fractionations are reported between the coexisting fluid and solid phases in 2D binary mixtures of big and small particles such as the squares and disks [57, 58]. It is very interesting that the mixing of squares and disks stabilizes the isotropic fluid with respect to the ordered phases, enhances the stability region of hexatic and tetratic phases and gives rise to fluid-solid and solid-solid demixing transitions [59]. It is also feasible that the mixing of particles with different shapes and sizes can induce demixing transitions even in the isotropic fluid phase [60]. In two dimensions the simplest binary mixture, which exhibits fluid-fluid demixing transitions is the nonadditive mixture of hard disks [61-67]. The positive nonadditivity is responsible for the fluid-fluid demixing transition, because it reduces the available room for both components upon mixing. It is showed that both the symmetric (the diameters of the components are the same) and the asymmetric hard disk mixtures can demix if the nonadditivity exceeds a minimum value [64, 
68, 69]. The additive three-dimensional (3D) hard body mixtures can exhibit both isotropicisotropic and nematic-nematic demixing transitions if the constituting particles are rod-like [60, 70-72]. Opposite to the 3D rod-rod mixtures, only nematic-nematic demixing transitions are found in binary mixtures of rod-like particles such as the rectangle-rectangle and ellipse-ellipse mixtures [73-76]. If the shapes of the particles are not very different in the disk-ellipse and ellipse-ellipse mixtures, the demixing transition and the segregation are not present, but both rotational and translational glass transitions may occur $[77,78]$.

In this work we examine the effects of varying shapes and sizes on the phase behaviour of two-dimensional hard convex objects using the scaled particle theory. We choose the superellipse shape, which provides a bridge between the elliptical and rectangular shapes. This shape allows us to consider new mixtures such as the ellipse-rectangle, where the shape of the ellipse can be tuned into the direction of rectangle and vice versa for rectangle. We concentrate only on the stability of isotropic, nematic and tetratic ordering and search for possible phase transitions such as the isotropic-nematic, isotropic-tetratic and nematic-nematic ones. As the inputs of the theory are the areas of the particles and the excluded area between two particles, we calculate the area of the superellipse and derive an algorithm for the excluded area between two superellipses. To understand the shape dependence of the observed segregations (isotropicnematic fractionation, nematic-nematic demixing) we make a link between the superellipse mixture and the nonadditive mixture of hard disks, where the nonadditivity parameter is the driving force of the fluid-fluid demixing. We finish our study with presenting a possible stabilization method of the low density tetratic order with mixing hard squares with long rodlike particles.

\section{Model}

In this work we examine the phase behaviour of some binary mixtures of hard 2D objects, where both the shape and size of the particles can be tuned easily. This $2 \mathrm{D}$ object is the hard superellipse with a semi-major axis length $(a)$ and a semi-minor axis length $(b)$. The equation of a superellipse depends also on the deformation parameter $(n)$ as follows

$$
\left(\frac{|x|}{a}\right)^{n}+\left(\frac{|y|}{b}\right)^{n}=1
$$


where $n \geq 2$ guaranties that the superellipse has a smooth curve and its tangent is well defined in its every point. For this reason, we deal only with this case in this paper. With increasing $n$, the shape of the particle can be tuned from the elliptical shape to the rectangular one. This opens the window to study a wider class of binary mixtures as the lengths and the shapes of both components can be varied independently. Using the diameter of the component one $\left(2 b_{1}\right)$ as a unit of the length, we have the following five independent molecular parameters: I) the aspect ratio of component $1\left(k_{1}=a_{1} / b_{1}\right)$, II) the aspect ratio of component $2\left(k_{2}=a_{2} / b_{2}\right)$, III) the diameter ratio $\left(d=b_{2} / b_{1}\right)$ and IV)-V) the exponents $\left(n_{1}\right.$ and $\left.n_{2}\right)$. The binary mixtures, which cannot be studied with the simple ellipse shape, are the followings: the square-square, square-ellipse, ellipse-rectangle and rectangle-rectangle mixtures. Note that this list is even longer if we replace one of the components or both components with superellipses having intermediate values of $n_{1}$ and $n_{2}$, which are between 2 (ellipse-limit) and infinity (rectanglelimit). It is also possible to study the binary mixture of hard superdisks if $a_{1}=b_{1}$ and $a_{2}=b_{2}$. Our experience is that we can get back the perfect rectangle shape and the area of the rectangle very accurately if $n=100$. For this reason $n_{1}$ and $n_{2}$ is always between 2 and 100 in this study. The components of a possible binary mixture is shown in Fig. 1, where the first component is an ellipse, while the second component is a superellipse.
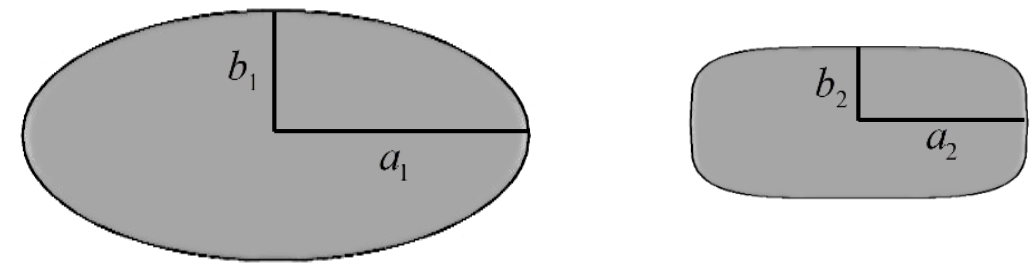

Figure 1: The particles of a possible superellipse mixture: a standard ellipse with $n_{1}=2$ (left) and a superellipse with $n_{2}=4$ (right) are shown.

\section{Theory}

We focus on the isotropic, tetratic and nematic phases of the binary mixture using the scaled particle theory (SPT). In the SPT approach [73], the free energy density of the binary mixture can be written as 


$$
\frac{\beta F}{A}=\sum_{i=1}^{2} \rho_{i}\left(\ln \rho_{i}-1+\sigma\left[f_{i}\right]\right)-\rho\left(\ln (1-\eta)+\frac{\eta}{1-\eta}\right)+\frac{1}{2(1-\eta)} \sum_{i, j=1}^{2} \rho_{i} \rho_{j}\left\langle\left\langle A_{\text {exc }}^{i j}\right\rangle\right\rangle,
$$

where $\beta=1 / k_{B} T$ is the inverse temperature, $A$ is the area of the surface, $\rho_{i}=N_{i} / A$ is the density of the component $i(i=1,2), N_{i}$ is the number of the particles of component $i$, $\rho=\rho_{1}+\rho_{2}$ is the total density, $\eta=\rho_{1} \tilde{a}_{1}+\rho_{2} \tilde{a}_{2}$ is the packing fraction, $\tilde{a}_{i}$ is the area of a particle of component $i$ and $A_{e x c}^{i j}$ is the excluded area between a particle of component $i$ and a particle of component $j$. In Eq. (2) we use the following short notations for the sake of brevity:

$$
\sigma\left[f_{i}\right]=\int_{0}^{2 \pi} f_{i}(\varphi) \ln f_{i}(\varphi) d \varphi
$$

and

$$
\left\langle\left\langle A_{e x c}^{i j}\right\rangle\right\rangle=\int_{0}^{2 \pi} \int_{0}^{2 \pi} f_{i}\left(\varphi_{1}\right) f_{j}\left(\varphi_{2}\right) A_{e x c}^{i j}\left(\varphi_{1}-\varphi_{2}\right) d \varphi_{1} d \varphi_{2}
$$

where $f_{i}$ is the orientational distribution function of the component $i$ and $\varphi_{i}$ is the orientation of a particle of component $i$. In the isotropic phase it is trivial to show that $f_{i}=\frac{1}{2 \pi}$, because $\int_{0}^{2 \pi} f_{i}(\varphi) d \varphi=1$. It can be shown that Eq. (2) reproduces the free energy of hard disk mixtures coming from SPT $[79,80]$. To do this we have to substitute the isotropic distributions $\left(f_{i}=\frac{1}{2 \pi}\right)$ and the excluded area between two disks $\left(A_{\text {exc }}^{i j}=\pi\left(a_{i}+a_{j}\right)^{2}\right)$ into Eq. (2). In order to find the tetratic and nematic solutions for the orientational distribution functions, we need to minimize the free energy with respect to $f_{i}$. This procedure results in a coupled integral equations for $f_{i}$, which can be written down as 


$$
f_{i}(\varphi)=\frac{\exp \left[-\frac{1}{1-\eta} \sum_{j=1}^{2} \rho_{j} \int_{0}^{2 \pi} A_{e x c}^{i j}\left(\varphi-\varphi_{1}\right) f_{j}\left(\varphi_{1}\right) d \varphi_{1}\right]}{\int_{0}^{2 \pi} \exp \left[-\frac{1}{1-\eta} \sum_{j=1}^{2} \rho_{j} \int_{0}^{2 \pi} A_{e x c}^{i j}\left(\varphi_{2}-\varphi_{1}\right) f_{j}\left(\varphi_{1}\right) d \varphi_{1}\right] d \varphi_{2}}
$$

The low density solutions of Eqs. (5) are isotropic. To find the tetratic and nematic solutions of Eqs. (5), we use nematic and tetratic trial functions for $f_{1}$ and $f_{2}$ as initial guesses, we do numerical integrations and use the iteration method. After having obtained the equilibrium distribution functions $f_{1}$ and $f_{2}$, we measure the extent of the orientational ordering of both components with 2D order parameters, which are defined as

$$
\begin{aligned}
& S_{2}(i)=\int_{0}^{2 \pi} f_{i}(\varphi) \cos (2 \varphi) d \varphi, \\
& S_{4}(i)=\int_{0}^{2 \pi} f_{i}(\varphi) \cos (4 \varphi) d \varphi,
\end{aligned}
$$

where $S_{2}(i)$ and $S_{4}(i)$ measure the nematic and tetratic ordering of the component $i$, respectively. Note that $S_{j}(i)=\int_{0}^{2 \pi} f_{i}(\varphi) \cos (j \varphi) d \varphi$ is the general order parameter of component $i$, where $j=2,4,6, \ldots$ etc.

In order to get some information about the orientational ordering transition, we perform a bifurcation analysis, which gives the stability limit of the isotropic phase with respect to the ordered phases such as the nematic and tetratic phases [81-83]. In the case of the second order phase transition this analysis provides either the isotropic-nematic or the isotropic-tetratic transition density. To perform this analysis we use the Fourier expansion of the excluded area, which can be written as

$$
A_{e x c}^{i j}\left(\varphi_{12}\right)=\sum_{k=0}^{\infty} A^{i j, k} \cos \left(k \varphi_{12}\right)
$$

where $A^{i j, k}$ is the Fourier component of the expansion and $\varphi_{12}=\varphi_{1}-\varphi_{2}$. Using Eq. (7) the integrals in Eq. (5) can be written as 
$\int_{0}^{2 \pi} A_{e x c}^{i j}\left(\varphi-\varphi_{1}\right) f_{j}\left(\varphi_{1}\right) d \varphi=\sum_{k=0}^{\infty} A^{i j, k} S_{k}(j) \cos (k \varphi)$

In the case of the nematic phase $S_{2}(j)$ starts to be nonzero first, while $S_{4}(j)$ is the first nonvanishing term for the tetratic ordering. Therefore $S_{2}(j)$ and $S_{4}(j)$ terms are taken into account at the IN and IT bifurcations, respectively, while the other order parameter terms can be considered to be zero. Denoting the first non-vanishing order parameter as $S_{l}(j)$ we get that

$$
f_{i}(\varphi)=\frac{\exp \left[-\frac{1}{1-\eta} \sum_{j=1}^{2} \rho_{j} A^{i j, l} S_{l}(j) \cos (l \varphi)\right]}{2 \pi}
$$

As $S_{l}(j)$ goes to zero at the bifurcation, we can perform a Taylor-expansion in Eq. (9) as $\exp (-\varsigma) \approx 1-\varsigma$. In the final step we multiply both sides of Eq. (9) with $\cos (l \varphi)$ and integrate out the $\varphi$ dependence to arrive into a set of coupled equations for the order parameters. We obtain the following two equations

$$
\begin{aligned}
& \left(1+\frac{\mathrm{c} \rho_{1} A^{11, l}}{2}\right) \mathrm{S}_{l}(1)+\left(\frac{\mathrm{c} \rho_{2} A_{e x c}{ }^{12, l}}{2}\right) \mathrm{S}_{l}(2)=0, \\
& \left(\frac{\mathrm{c} \rho_{1} A_{e x c}{ }^{21, l}}{2}\right) \mathrm{S}_{l}(1)+\left(1+\frac{\mathrm{c} \rho_{2} A_{e x c}^{22, l}}{2}\right) \mathrm{S}_{l}(2)=0,
\end{aligned}
$$

where $c=1 / 1-\eta$. These equations can be written down as a product of a matrix and a vector, where the matrix elements are the prefactors of the order parameters, while the order parameters constitute the vector. To have a non-trivial solution of Eq. (10) the determinant of the matrix must be zero. This produces the following bifurcation equation

$$
\left(1+\frac{c \rho_{1} A^{11, l}}{2}\right)\left(1+\frac{c \rho_{2} A^{22, l}}{2}\right)-\frac{c^{2} \rho_{1} \rho_{2} A^{12, l} A^{21, l}}{4}=0
$$

We solve this equation numerically for a given molecular parameters $\left(k_{1}, k_{2}, d, n_{1}\right.$ and $\left.n_{2}\right)$ and mole fractions $\left(x_{i}=N_{i} / N\right)$ to get the bifurcation density $(\rho)$ and packing fraction $(\eta)$ as $\rho_{i}=x_{i} \rho$. 
In the case of first-order phase transitions we need to know the chemical potentials of each components and the pressure of the system. These quantities come from the following equations:

$$
\begin{gathered}
\mu_{i}=\frac{\partial\left(\frac{F}{A}\right)}{\partial \rho_{i}}, \\
P=-\frac{F}{A}+\sum_{i=1}^{2} \mu_{i} \rho_{i} .
\end{gathered}
$$

Applying the above equations we can determine the equation of the state of the system and study the stability of the isotropic, nematic and tetratic phases of the binary mixture. The coexisting densities of a phase transition can be calculated using $\mu_{1}^{\alpha}=\mu_{1}^{\beta}, \mu_{2}^{\alpha}=\mu_{2}^{\beta}$ and $P^{\alpha}=P^{\beta}$, where the phases $\alpha$ and $\beta$ are in equilibrium.

In order to solve Eqs. (5) and (11) at a given density and composition, we need to know the molecular areas and the excluded areas of the superellipses. It can be proved that $\tilde{a}_{i}=\frac{a_{i} b_{i}}{n_{i}} 2^{2\left(1-\frac{1}{n_{i}}\right)} \sqrt{\pi} \frac{\Gamma\left(\frac{1}{n_{i}}\right)}{\Gamma\left(\frac{1}{n_{i}}+\frac{1}{2}\right)}$ is the area of a superellipse of the component $i$, where $\Gamma$ is the Gamma function. In the ellipse-limit $\left(n_{i}=2\right)$ we can see that $\tilde{a}_{i}=\pi a_{i} b_{i}$, while $\tilde{a}_{i}=4 a_{i} b_{i}$ is in the rectangle-limit $\left(n_{i} \rightarrow \infty\right)$. The excluded area between a particle $i$ with orientation $\varphi_{i}$ and a particle $j$ with orientation $\varphi_{j}$, when the angle between the main axes of these two particles is $\varphi_{i j}=\varphi_{i}-\varphi_{j}$, can be written as follows

$$
\begin{aligned}
& A_{e x c}^{i j}\left(\varphi_{i j}\right)=\tilde{a}_{i}+\tilde{a}_{j} \\
& +2 \int_{0}^{\pi}\left(\left[\frac{d r_{j}}{d \theta_{j}} \cos \left(\theta_{j}+\varphi_{i j}\right)-r_{j} \sin \left(\theta_{j}+\varphi_{i j}\right)\right] \sin \theta_{i}-\left[\frac{d r_{j}}{d \theta_{j}} \sin \left(\theta_{j}+\varphi_{i j}\right)+r_{j} \cos \left(\theta_{j}+\varphi_{i j}\right)\right] \cos \theta_{i}\right) r_{i} d \theta_{j} \\
& \text { where } r_{i}=\left[\left(\frac{\left|\cos \theta_{i}\right|}{a_{i}}\right)^{n_{i}}+\left(\frac{\left|\sin \theta_{i}\right|}{b_{i}}\right)^{n_{i}}\right]^{\frac{-1}{n_{i}}} \text {. Note that } \theta_{i} \text { has a complicate dependence from } \theta_{j} \text {. }
\end{aligned}
$$

Therefore we present the details of the excluded area calculation in the Appendix. As the 
diameter of component one $\left(2 b_{1}\right)$ is taken to be the unit of the length, $P^{*}=4 b_{1}^{2} \beta P$ is the dimensionless pressure and $A_{e x c}^{*}=A_{e x c} /\left(2 b_{1}\right)^{2}$ is the dimensionless excluded area.

\section{Results}

We start to show how the hard superellipse changes its shape from the ellipse to rectangle with increasing the deformation parameter (n). We can see in Fig. 2(a) that the

(a)

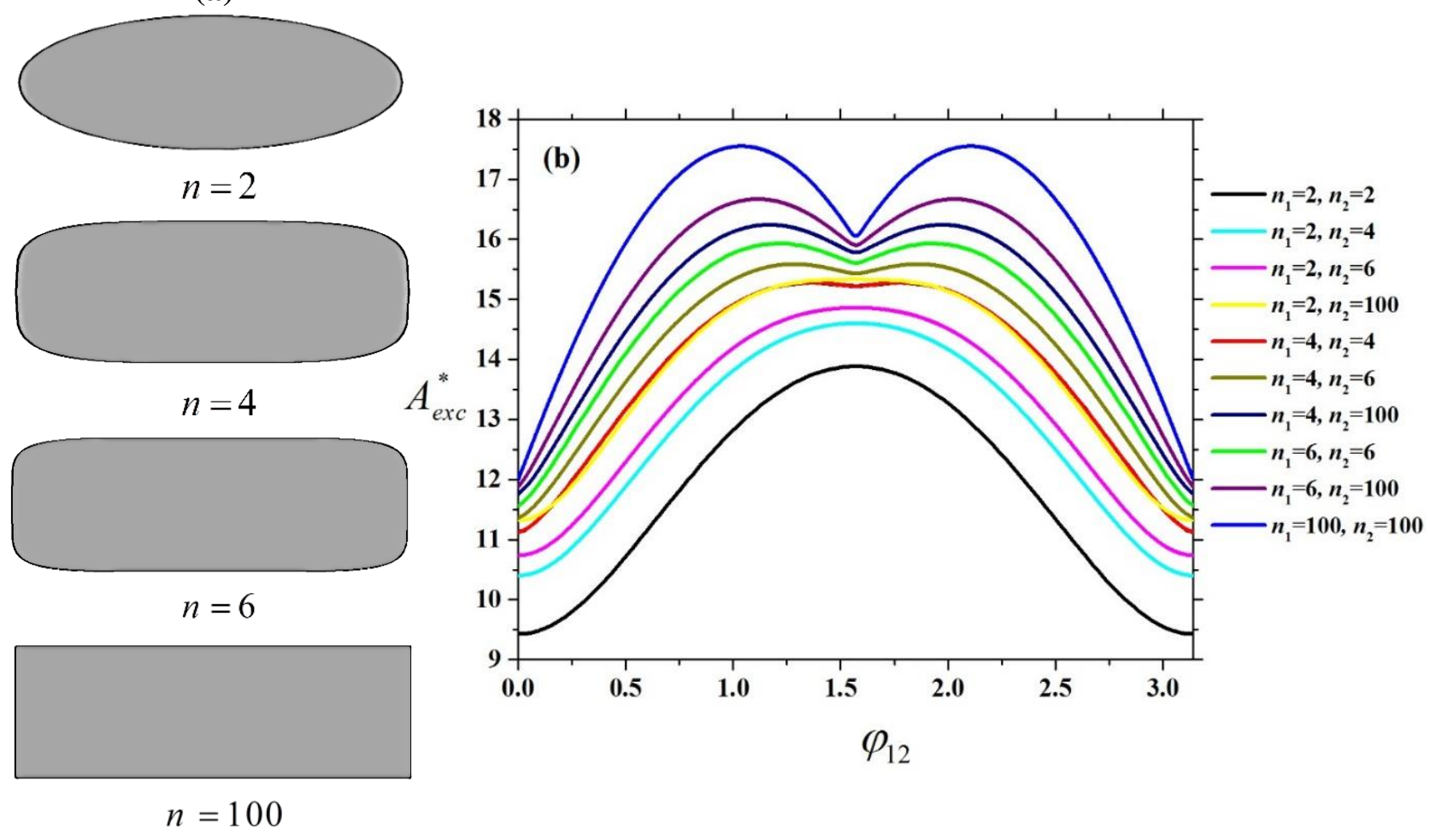

Figure 2: Deformation parameter dependence of the shape and the excluded area of hard superellipses at $k=3$. The following cases are shown: $n=2,4,6$ and 100. (a) The effect of varying deformation parameter $(n)$ on the shape of the superellipse. (b) The excluded area between two hard superellipses with deformation parameters $n_{1}$ and $n_{2}$ as a function of $\varphi_{12}$, where $\varphi_{12}$ is the angle between the major axes of two particles. All possible combinations of $n(2,4,6$ and 100) are shown together. The lengths of the short $(2 a)$ and long $(2 b)$ axes of the superellipse are kept fixed.

particle becomes more and more rectangular with increasing $n$. The case of $n=2$ corresponds to an ellipse, while $n=100$ almost to a rectangle. Practically, we find that the area of the rectangle is identical with that of a superellipse if $n=100$. The excluded area between two hard superellipses for all possible combinations of $n(2,4,6$ and 100) is shown as a function of 
$\varphi_{12}$ angle in Fig. 2(b). With increasing $n_{1}$ and $n_{2}$ the excluded area is shifted to the direction of higher values and a local minimum arises at $\varphi_{12}=\pi / 2$. It is interesting that $n_{1}=n_{2}=4$ case is an intermediate shape between ellipse and rectangle as the local minimum just appears at $\varphi_{12}=\pi / 2$. We can also see that the ellipse shape is the best for nematic ordering as this shape provides the highest excluded area gain with parallel alignment.

An interesting system to study is the binary mixture of short and long rods which exhibits strong demixing behaviour in three dimensions [84-86]. Fig. 3 shows together the phase diagrams of some binary mixtures of short and long superellipses for $k_{1}=5, k_{2}=15$ and $d=1$ in the reduced pressure-composition plane. The area ratio of long and short superellipses is located between $3 \pi / 4 \approx 2.36$ (the case of short rectangle and long ellipse) and $12 / \pi \approx 3.82$ (the case of short ellipse and long rectangle), which means that all possible mixtures are moderately asymmetric in area. We can see first-order and second-order isotropic-nematic $(I-N)$ phase transitions, fractionations, re-entrance phenomena and nematic-nematic $(N-N)$ demixing transitions with or without lower critical points. As the phase diagram changes a lot with the deformation parameters $\left(n_{1}\right.$ and $\left.n_{2}\right)$, the shape has big impact on the coexisting mole fractions at both low and high pressures. It can be seen clearly that the $N-N$ demixing transition is the weakest and postponed to higher pressures in the case of the rectangle-rectangle mixture, while it becomes very stable if the long component is changed to be ellipse (see Fig. 3(a)). The $I-N$ phase transition exhibits unusual behaviour as it changes from second-order to first-order with increasing pressure. The first-order $I-N$ transition is very weakly fractionated for the short ellipse-long rectangle mixture, while it shows very big difference in the coexisting mole fractions for the short rectangle-long ellipse mixture. It can be also seen that the $N-N$ demixing transition terminates in a lower critical point for rectangle-rectangle, ellipse-ellipse and ellipserectangle mixture, while a second-order $I-N$ transition bifurcates and a first-order $N-N$ transition merges from a first-order $I-N$ coexistence in the case of the rectangle-ellipse mixture. To get further insight into the behaviour of $I-N$ and $N-N$ transitions, we consider the short superellipselong ellipse mixture in Fig. 3(b), where the deformation parameter of the superellipse $\left(n_{1}\right)$ varies between 2 and 6 . It can be seen that the increasing $n_{1}$ widens the coexistence regions of both $I-N$ and $N-N$ transitions. Comparing with the results of the ellipse-ellipse mixture, we can observe that a critical endpoint and an upper $N-N$ critical point emerge for $n_{1}=4$, while the lower $N-N$ critical point occurs at a lower pressure. At $n_{1}=6$ the lower and the upper $N-N$ critical pressures are not present due to the stabilisation of the $N-N$ demixing transition at low 
pressures. The merge of upper and lower critical points takes place around $n_{1}=5$, which is not shown in this study. We can also observe strong re-entrant phenomena in Fig. 3, because an isotropic-nematic-isotropic-nematic phase sequence can take place with increasing pressure at some compositions. The reentrance of the isotropic phase with increasing pressure becomes more pronounced with growing $n_{1}$ as the mole fraction gap of the $I-N$ transition widens with $n_{1}$. For example we can observe an isotropic phase which is in coexistence with a nematic one at intermediate pressures if $0.45<x_{1}<0.82$ for $n_{1}=6$, while this phase sequence occurs only in the interval of $0.73<x_{1}<0.83$ for $n_{1}=2$. It is interesting that the reentrant feature of the isotropic phase was also observed in the lattice gas of 2D short and long hard rods [87]. Regarding the stability of the nematic ordering we show the pressure vs. composition curve at $\eta=0.8$ for the ellipse-ellipse mixture in Fig. 3, which is dictated by the simulation results obtained for monodisperse hard ellipse [40] and hard discorectangle [33] systems, where the nematic-solid transitions are detected at $\eta \sim 0.8$. In our binary mixture the different sizes and shapes of the components work against the crystallisation, i.e. demixed solid phases are expected to occur at higher packing fractions and pressures. We note the phase diagrams of 3D binary mixtures of hard rods are similar to those of hard superellipses with some important differences: 1) an isotropic-isotropic demixing is also present 2) the $I-N$ transition is always
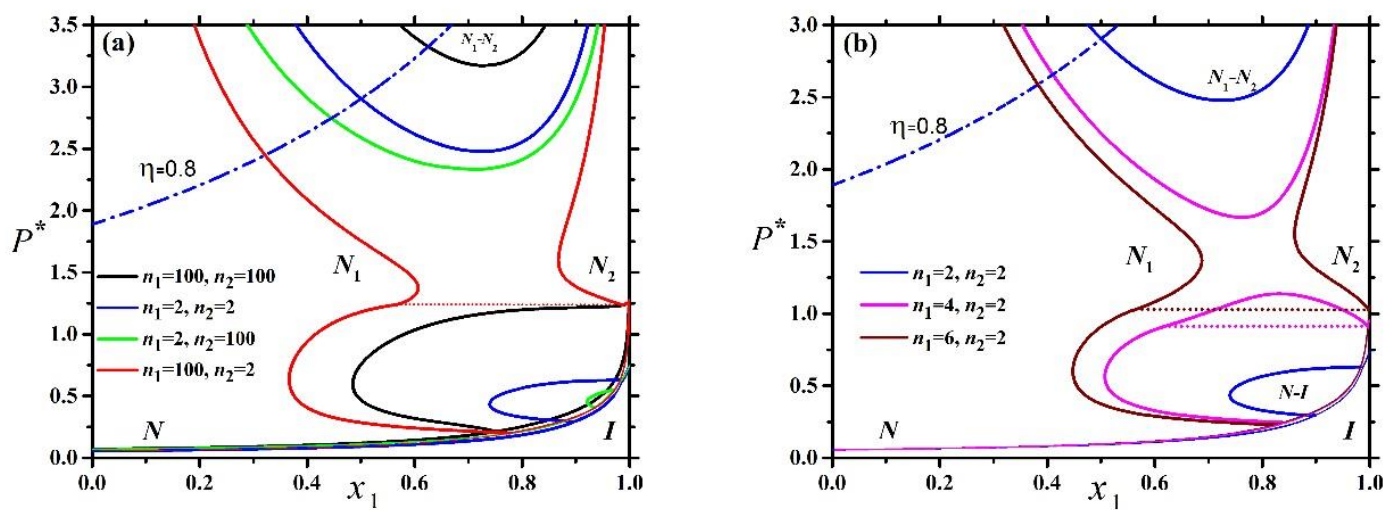

Figure 3: The effect of varying shape on the phase diagram of the binary mixture of short and long rods. The aspect ratios of short and long rods and the diameter ratio are chosen as follows: $k_{1}=5, k_{2}=15$ and $d=1$. Figure (a) shows the phase diagrams of rectangle-rectangle, ellipse-ellipse, ellipse-rectangle and rectangle-ellipse mixtures, while Figure (b) presents the effect of varying deformation parameter of the short superellipses on the phase diagram of superellipse-ellipse mixture. The phase diagrams are presented in pressure-composition plane where $x_{1}$ is the mole fraction of short rods. $I$ and $N$ denote the isotropic and nematic phases, respectively. The horizontal dashed line represents the $I-N$ coexistence at the critical endpoint. The dashed-dotted line indicates the location of the high density region of the phase diagram, which is calculated for ellipse-ellipse mixture at $\eta=0.8$. 
first-order, 3) the re-entrant feature is weak and 4) the $I-N$ fractionation is stronger in the $3 \mathrm{D}$ rod-rod mixtures [70-72, 76, 88-91].

In the following, our aim is to understand the results of Fig. 3 with the introduction of new quantities, which characterize the demixing tendency of the superellipse mixture. For this purpose it is worth mapping the binary mixture of hard superellipses into a nonadditive binary mixture of hard disks, where the nonaddivity parameter is responsible for the fluid-fluid demixing transition. In more details, the binary mixture of nonadditive hard disks is described with three parameters: $\sigma_{1}$ and $\sigma_{2}$ are the diameters of the components and $\Delta$ is the nonadditivity parameter effecting only the unlike contact distance, $\sigma_{12}$, via

$$
\sigma_{12}=\frac{\sigma_{1}+\sigma_{2}}{2}(1+\Delta)
$$

It was shown that $\Delta$ must be high enough $(>0.2)$ to obtain fluid-fluid demixing transitions in the binary mixture of hard disks [69]. By equating the like $\left(A_{e x c}^{11}, A_{e x c}^{22}\right)$ and unlike $\left(A_{e x c}^{12}\right)$ excluded areas of the nonadditive hard disk mixture and those of the hard superellipse mixtures, we can get the corresponding molecular parameters of the binary hard disk mixture $\left(\sigma_{1}, \sigma_{2}\right.$ and $\Delta)$ in the perfectly ordered nematic (the particles are parallel) and isotropic phases. In this way we can derive that the nematic nonadditivity parameter is given by

$$
\Delta_{N}=2 \frac{\sqrt{A_{\text {exc }}^{12}\left(\varphi_{12}=0\right)}}{\sqrt{A_{\text {exc }}^{11}\left(\varphi_{12}=0\right)+\sqrt{A_{\text {exc }}^{22}\left(\varphi_{12}=0\right)}}}-1,
$$

where the like and unlike excluded areas are evaluated at parallel configurations. In the isotropic phase we can get the same type of formula, but the isotropically averaged excluded areas must be used in Eq. (16), i.e.

$$
\Delta_{I}=2 \frac{\sqrt{\left\langle\left\langle A_{\text {exc }}^{12}\right\rangle\right\rangle}}{\sqrt{\left\langle\left\langle A_{\text {exc }}^{11}\right\rangle\right\rangle+\sqrt{\left\langle\left\langle A_{\text {exc }}^{22}\right\rangle\right\rangle}}}-1
$$

where $f_{i}(\varphi)=1 / 2 \pi$ has to be substituted into Eq. (4). One can show that $\Delta_{N}$ is still negative when the unlike excluded area equal with the geometric mean of the like excluded areas, i.e. $A_{e x c}^{12}=\sqrt{A_{e x c}^{11} A_{e x c}^{22}}$, while it turns to be positive for the arithmetic mean $\left(A_{e x c}^{12}=\left(A_{e x c}^{11}+A_{e x c}^{22}\right) / 2\right)$. 
This shows that the positive value of $\Delta_{N}$ corresponds to the situation when the mixing of the components is not favorable for at least one of the components because $A_{e x c}^{12}>\min \left(A_{e x c}^{11}, A_{e x c}^{22}\right)$. With increasing $\Delta_{N}$ it may happen that the mixing reduces the available room for both components, which is not favourable for the mixture.

Fig. 4 illustrates the demixing tendencies of the superellipse-superellipse mixtures in the isotropic and nematic phases including those systems, which are discussed in Fig. 3. It can be seen that the isotropic demixing tendency is very weak for the mixtures as $\Delta_{I}$ is negative or just slightly higher than zero. Opposite to this, the parallel nematic ordering has positive $\Delta_{N}$, which indicates that the components of the mixture do not like to be mixed together as the unlike excluded area reduces the available room for the particle in a higher extent than the like terms. In agreement with Fig. 3 we can see that the highest values of $\Delta_{I}$ and $\Delta_{N}$ belong to the rectangle-ellipse mix ture, which has the widest $I-N$ and $N-N$ coexistence regions. Fig. 4 explains also why the weak $I-N$ transition is accompanied by a relatively strong $N-N$ demixing transition in the ellipse-rectangle mixture (see Fig. 3). Namely, $\Delta_{I}$ is the smallest, while $\Delta_{N}$ is almost the largest for the ellipse-rectangle mixture in comparison with the other mixtures. The weakest $N$ $N$ demixing is observed in the rectangle-rectangle mixture for which $\Delta_{N}$ is the lowest. These results show that $\Delta_{N}$ informs us about the $N-N$ demixing tendency, while $\Delta_{I}$ is relevant for the $I-N$ transition. To find wide coexistence regions in the $I-N$ and $N-N$ transitions, we observe that both $\Delta_{I}$ and $\Delta_{N}$ should be high enough. Therefore stronger $I-N$ and $N-N$ transitions can be observed for the systems with $d$ in the interval of $0.25<d<1$, because both nonadditivity
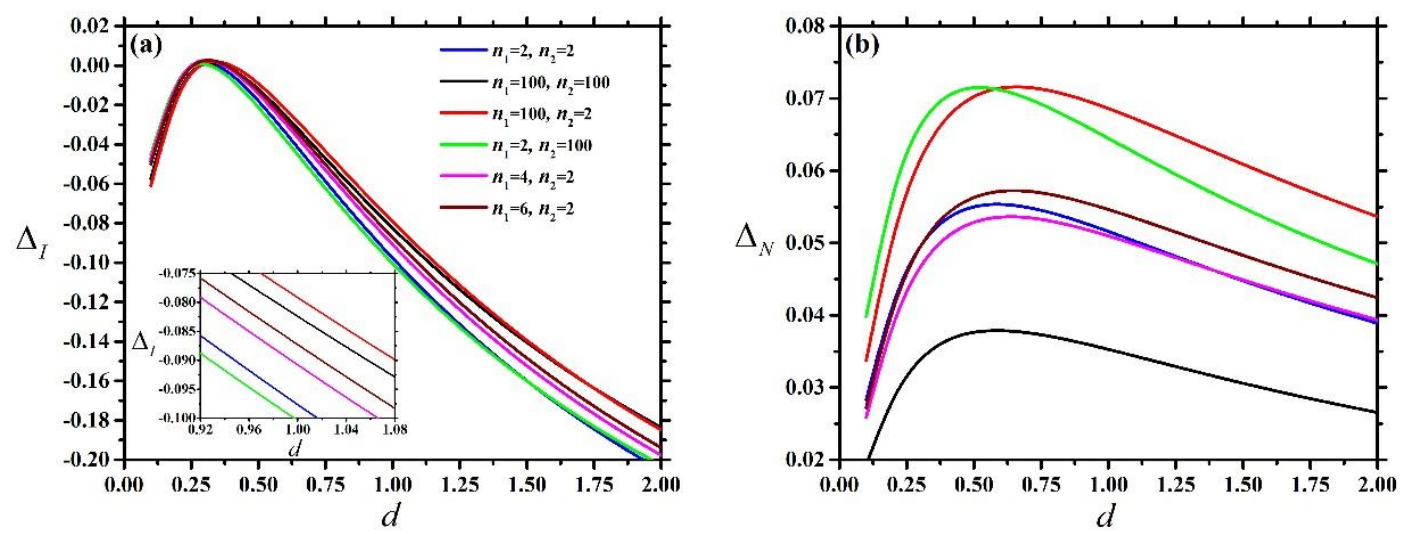

Figure 4: Isotropic and nematic nonadditivity parameters $\left(\Delta_{I}\right.$ in (a) and $\Delta_{N}$ in (b)) versus diameter ratio $(d)$ of some binary mixtures of hard superellipses with $k_{1}=5$ and $k_{2}=15$. The inset enlarges a small part of $\Delta_{I}-d$ curves in (a). The color key is the same in (a) and (b). 
parameters are higher than $\Delta$ values of the case of $d=1$. We observe that the coexistence regions are narrower for $d>1$.

Our search for finding the highest values of the nonadditivity parameters has resulted in a binary mixture, where the components are the disks and needles. In this regard Fig. 5 demonstrates that lowering $k_{1}$ results in higher values for both $\Delta_{N}$ and $\Delta_{I}$ if the second component is almost needle. We find that the highest value of $\Delta_{I}$ is $\sqrt{2 /(2 \sqrt{2}-1)}-1 \approx 0.046$ in the disk-needle mixture, which is not high enough to induce the isotropic-isotropic (I-I) demixing transition. However, the high values of $\Delta_{I}$ and $\Delta_{N}$ give rise to very strong fractionations (wide coexistence gap) occurring between the coexisting isotropic and nematic phases. To observe the $I-I$ demixing, we believe that much higher values of $\Delta_{I}$ should be required, which is not possible to achieve with the present superellipse model. Therefore we are quite sure that the $I-I$ demixing transition cannot be found in binary superellipse systems using the scaled particle theory.
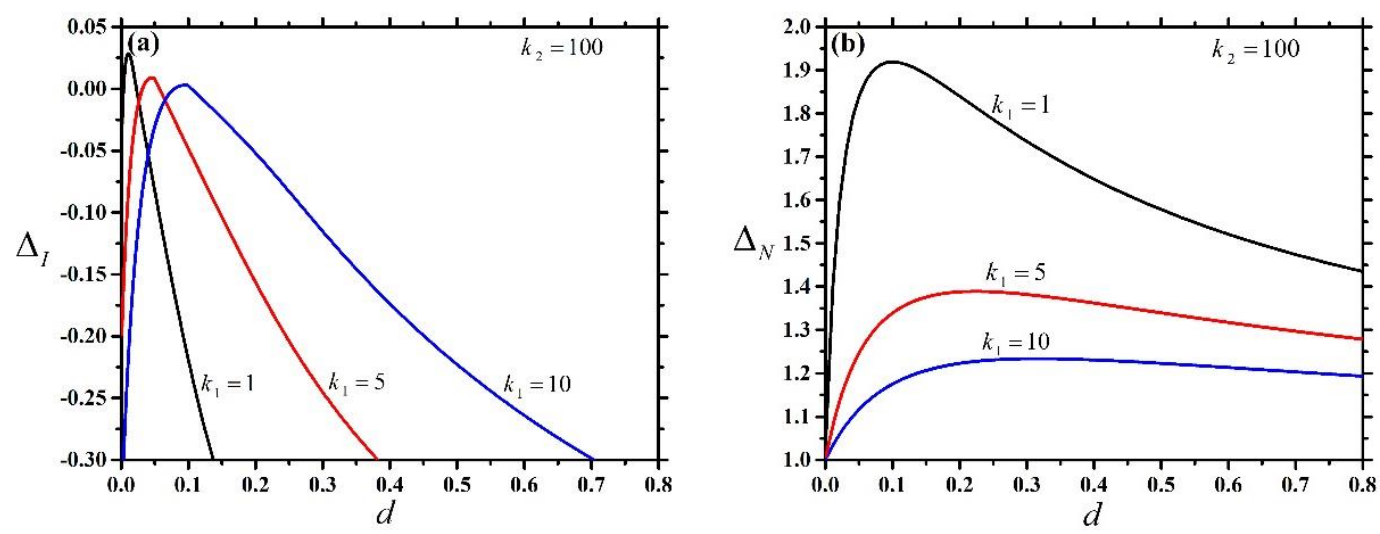

Figure 5: Isotropic and nematic nonadditivity parameters $\left(\Delta_{I}\right.$ in (a) and $\Delta_{N}$ in (b)) versus diameter ratio $(d)$ of some binary mixtures of superellipses, where $k_{2}=100$ and $k_{1}=1,5$ and 10. Both $n_{1}$ and $n_{2}$ are chosen to be two (ellipse-ellipse mixture).

As a demonstration we show the phase diagrams of two systems, which belong to Fig. 5. One is chosen to have high $\Delta_{N}$ value (Fig. 6(a)), while the other has almost maximal $\Delta_{I}$ value (Fig. 6(b)). The first system is weakly, while the second one is extremely asymmetric as the area ratio of the long and short ellipses is 0.8 in Fig. 6(a) (where $k_{1}=5, k_{2}=100$ and $d=0.2$ ) and 0.05 in Fig. 6(b) (where $k_{1}=5, k_{2}=100$ and $d=0.05$ ). We can see that $\Delta_{N}$ has effect even on the $I-N$ transition, too, because $\Delta_{I}$ is very low for the system of Fig. 6(a). Its effect manifests in the 
very strong fractionation between the coexisting isotropic and nematic phases. The effect of $\Delta_{I}$ is weaker even if it is positive for the system of Fig. 6 (b). The relatively high value of $\Delta_{I}$ is not accompanied even by the first-order $I-N$ transition, because $\Delta_{N}$ value is low in this system. Even the $N-N$ demixing curve is postponed to the direction of very high pressures in this case. Note that the solid phase cannot be stable in Fig. 6, because $\eta$ is far from the close packing value even at the highest examined pressures.
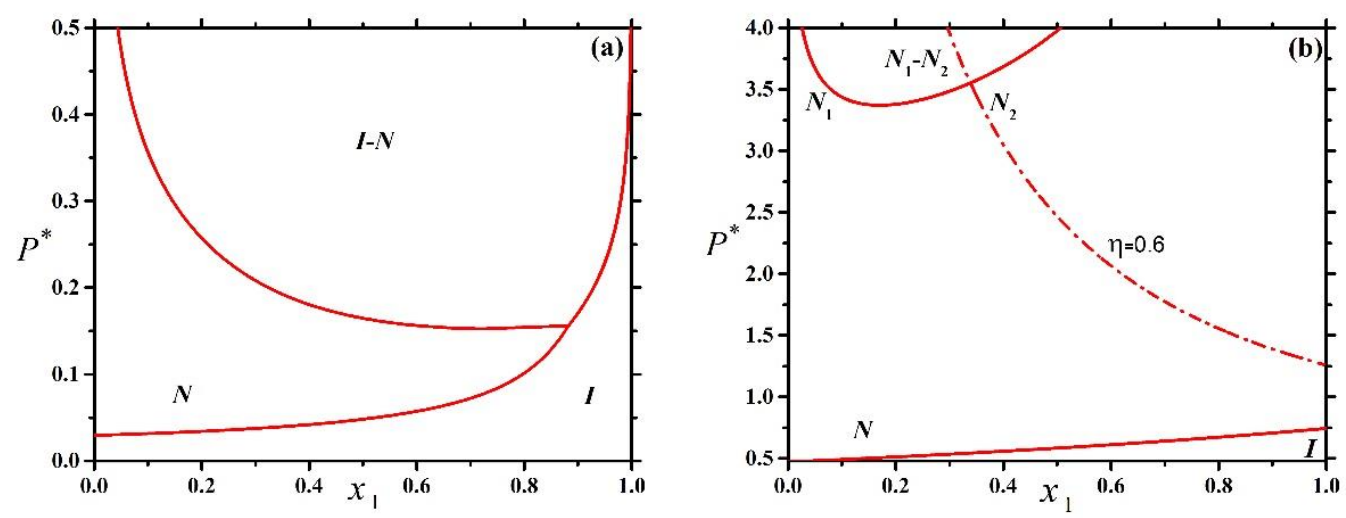

Figure 6: Phase diagrams of binary mixtures of hard ellipses $\left(n_{1}=n_{2}=2\right)$ where $k_{1}=5, k_{2}=100$. The following cases are considered: (a) $d=0.2$. and (b) $d=0.05$. The phase diagram is presented in pressurecomposition plane. $I$ and $N$ denote the isotropic and nematic phases, respectively. The dashed-dotted line indicates the location of $\eta=0.6$ values, which separates the high and low density regions.

We also consider the possibility of the tetratic ordering in the binary mixture of hard superellipses. The tetratic phase is characterized by $S_{2}=0$ and $S_{4} \neq 0$. This is different from the nematic phase, where $S_{2} \neq 0$. This means that the orientational distribution function is periodic by $\pi / 2$ in the case of the tetratic phase, while the nematic has the period of $\pi$. It has been observed that the tetratic phase can be stable in the fluid of weakly anisotropic hard rectangles $(k \geq 1)$, including the hard squares, too [14]. However, the tetratic phase can be stable only in very dense systems, where the packing fraction is more than about $0.75[17,18$, 23]. To find the tetratic ordering at lower packing fractions we mix the hard squares with long superellipses. The orientationally ordered phase must be nematic for the long particles, but it remains tetratic for the squares due to symmetry reasons. We show the phase diagram of some mixtures of squares and superellipses in Fig. 7. These mixtures are very asymmetric as the area ratio of the superellipse and the square is between $10 \pi$ and 40 . In all cases the effect of adding hard squares to the sea of rod-like particles destabilizes the $I-N$ transition by shifting the $I-N$ 
transition to the direction of higher pressures. This is not surprising as the squares do not form nematic phase, but they have isotropic-tetratic phase transition at $\eta \approx 0.86$ and $P^{*} \approx 53.6$. The destabilization effect of the squares on the $I-N$ curves seems to be weak up to $x_{1}=0.7$ for all cases, while it becomes very strong for $x_{1}>0.9$. This indicates that the squares can stay in the tetratic order in a wide range of the composition. The other interesting feature of Fig. 7 is that the $N-N$ demixing transition occurs in the square rich region of the composition $\left(x_{1}>0.5\right)$. This means that the squares induces a depletion attraction between the nematically ordered superellipses. This transition can be considered as a "vapour-liquid" transition of oriented superellipses in the sea of hard squares. It is also interesting that the $N-N$ transition terminates in a lower critical pressure, the second-order $I-N$ transition terminates in a critical endpoint and the first-order $I-N$ transition emerges at pressures higher than the largest pressure of the $N-N$ demixing. The $N-N$ and $I-N$ transitions seem to be stable because the pressure is very high at any composition for $\eta=0.8$. The system which has the widest stability region of the nematic phase is the square-rectangle mixture, because the region of the $N-N$ demixing is located at

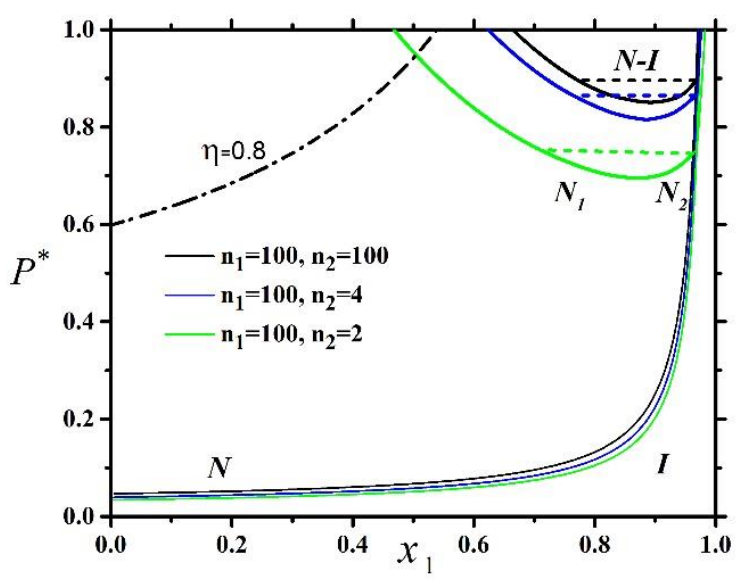

Figure 7: The effect of deformation parameter $\left(n_{2}\right)$ on the phase diagram of square-superellipse mixture. The first component is always hard square $\left(k_{1}=1\right)$, while the second one is superellipse $\left(k_{2}=10\right)$. The diameter ratio $(d)$ is chosen to be 2 . The phase diagram is presented in pressure-composition plane, where $x_{1}$ denotes the mole fraction of hard squares. $I$ and $N$ denote the isotropic and nematic phases, respectively. The horizontal dashed line represents the $I-N$ coexistence at the critical endpoint. The dashed-dotted line indicates the location of $\eta=0.8$ values for the square-rectangle mixture.

higher pressures than the $N-N$ regions of other two cases. The extent of the tetratic ordering of the hard squares surrounded by the nematically ordered long rectangles is presented in Fig. 8 
at some pressures, where the tetratic order parameter of the squares and the corresponding packing fraction are shown as a function of the mole fraction of the squares $\left(x_{1}\right)$ up to the $I-N$ bifurcation point. We can see that the long rods acts as an external orienting field on the squares by forcing them to be parallel or perpendicular to the nematic director. However, the strength of the orienting field increases slowly with the pressure as $S_{4}$ does not exceed 0.2 even at $P^{*}=0.5$ (Fig. 8 (a)). We can also see that the packing fraction of the isotropic-tetratic transition of hard squares $\left(\eta_{I T} \approx 0.86\right)$ is always larger than the corresponding packing fraction of the mixture at the pressures shown in Figure 8 (b). This indicates that the tetratic ordering can be stabilized at lower packing fractions in the mixture of squares and rectangles. This tetratic ordering is weak, but it can exist at low packing fractions (even at $\eta=0.4$ ).
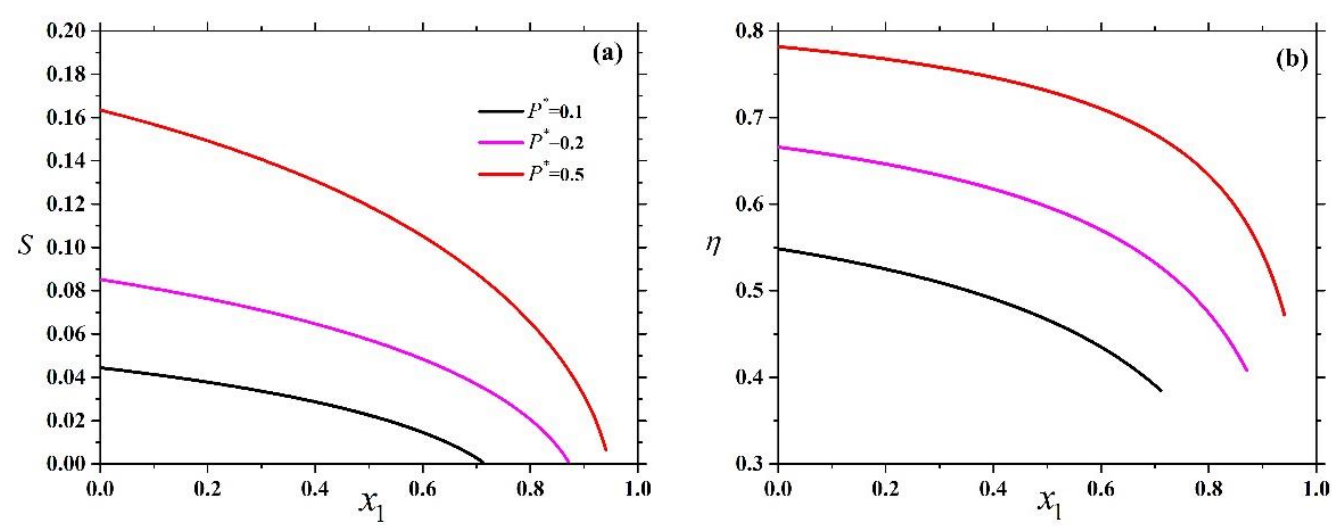

Figure 8: Tetratic ordering of hard squares in the mixture of hard squares and hard rectangles. Tetratic order parameter (a) and packing fraction (b) as a function of composition at some pressures, where the rectangles form nematic phase. The molecular properties of the mixture: $k_{1}=1, k_{2}=10, d=2$ and $n_{1}=n_{2}=100$.

Finally we have examined the effect of varying diameter ratio on the nonadditivity parameters. At the above examined case presented in Figs. 7 and 8, both $\Delta_{I}$ and $\Delta_{N}$ are low, which is the reason of having weak first-order $N-N$ demixing and second-order $I-N$ transitions in the mixture. Fig. 9 suggests that the $N-N$ demixing and the $I-N$ transition become stronger with lowering $d$, which shrinks the stability region of the nematic phase. With increasing $d$ the demixing tendency weakens, which makes a wider room for the nematic phase. However, the squares becomes even smaller with respect to the rectangles, so the tetratic ordering also weakens. Therefore the stabilization of the tetratic phase at low packing fractions is feasible in such square-rectangle mixtures, where the rectangles are long enough to form the nematic phase at low packing fractions and the squares are not very small with respect to the rectangles. Fig. 9 confirms the observed trends with changing the shape of the rods in Fig. 7, too, because 
$\Delta_{N}$ is largest for the square-ellipse mixture, which has the strongest $N-N$ demixing transition and high pressure first-order $I-N$ transition with strong segregation. We can also see that the lowest $N$ - $N$ demixing tendency $\left(\Delta_{N}\right)$ belongs to the square-rectangle mixture for all values of $d$. This proves that the square-rectangle mixture is the best system for the observation of tetratic order at any value of $d$. We have also examined the aspect ratio $\left(k_{2}\right)$ dependence of $\Delta_{N}$. In general $\Delta_{N}$ becomes larger with increasing $k_{2}$, i.e. the $N-N$ critical pressure decreases and the nematic-nematic demixing becomes stronger. However, this does not shrinks the stability region of the nematic phase, because the $I-N$ transition pressure is also shifted to lower values with increasing $k_{2}$. For example, we have observed very weak tetratic ordering of the squares in the nematic host with $k_{2}=100$ even at $\eta \approx 0.07$. This result is very promosing to detect tetratic ordering at very low densities in colloidal and vibrated granular mixtures.
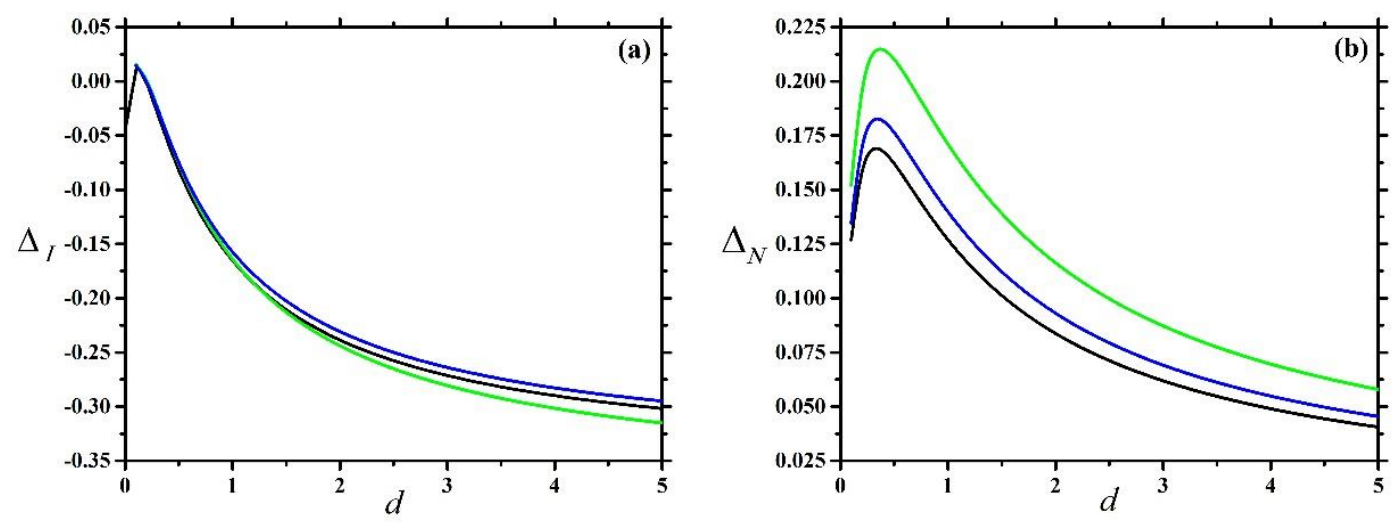

Figure 9: Isotropic and nematic nonadditivity parameters $\left(\Delta_{I}\right.$ in (a) and $\Delta_{N}$ in (b)) versus diameter ratio $(d)$ of some binary mixtures of hard squares and superellipses with $k_{1}=1, k_{2}=10$ and $n_{1}=100$. The deformation parameter of the second component changes between ellipse and rectangle shapes as follows: $n_{2}=2$ (green), 4 (blue) and 100 (black).

\section{Conclusion}

We have presented the results of the scaled particle theory for binary mixtures of hard superellipses, where the particles are staying in a plane. Using superellipse shape it is managed to interpolate between ellipse and rectangle shapes with changing the deformation parameters ( $n_{1}$ and $n_{2}$ ). This model helped us to examine the effects of size and shape disparities on the stability of isotropic and orientationally ordered (nematic and tetratic) phases. We have found first- and second-order $I-N$ and first-order $N-N$ phase transitions with strong fractionation 
between the coexisting phases. In addition to these transitions, a weak tetratic ordering of hard squares can be observed in the nematic host of long particles.

The observed phase diagrams are very sensitive to the shape of the constituting particles, i.e. a small change in the shape may result in a big change in the phase diagram. For example tuning the shape of the particles between the limiting rectangle and ellipse shapes, the $I-N$ and $N-N$ transition properties of the mixture of short and long particles change dramatically even if the aspect ratios and the diameter ratio are kept fixed. The $I-N$ and $N-N$ coexistence regions become very wide in short rectangle-long ellipse mixture, while the $I-N$ transition is only weakly fractionated and the $N-N$ transition is postponed to the direction of higher pressures in short ellipse-long rectangle mixture. As the $N-N$ demixing and the $I-N$ fractionation are related to the unlike (ellipse-rectangle) interactions, our results show that the unlike excluded area has big impact on the phase behavior of the binary mixture of short and long particles. With changing only deformation parameter of the short particles $\left(n_{1}\right)$ from 2 to 6 we can find three different types of phase diagrams: 1 ) weakly fractionated $I-N$ transition and $N-N$ demixing with lower critical point, 2) fractionated $I-N$ transition and $N-N$ demixing with both lower and upper critical points and 3) strongly fractionated $I-N$ transition and $N-N$ demixing without critical points. To explain the observed trends we mapped the binary mixture of superellipses into a binary mixture of nonadditive hard disks to obtain effective isotropic and nematic nonadditivity parameters $\Delta_{I}$ and $\Delta_{N}$. Using these parameters we can explain the observed trends in the change of the phase diagrams. To induce fractionation between the coexisting phases $\Delta_{N}$ must be positive, while $\Delta_{I}$ can be even negative. Our conclusion is that higher $\Delta_{N}$ and $\Delta_{I}$ give rise to stronger segregation between the components. However the predictive power of these nonadditivity parameters proved to be limited because $\Delta_{N}$ should depend on the degree of the nematic ordering, too. In the present form $\Delta_{N}$ can be useful to predict demixing transition at very high pressure, where the nematic order is very strong. To find such binary mixtures where the $I-N$ transition is always second-order or both first- and second-order $I-N$ transitions are present, we think that the full phase diagram calculation is inevitable. With tracking the critical endpoint and the upper and lower critical points it is possible to construct global phase diagram of binary mixtures of 2D hard bodies.

We have also looked for the possibility of a demixing transition, where the coexisting phases are isotropic. To do this we have searched for the maximum of $\Delta_{I}$ using a simplex algorithm. The maximum is located in the system of needle-disk mixture, where 
$\Delta_{I}=\sqrt{2 /(2 \sqrt{2}-1)}-1$. This value proved to be too small to induce the $I-I$ demixing transition. However this value is enough high to produce very strong fractionation between the coexisting isotropic and nematic phases. These results are consistent with the prediction of [63], where the possibility of $I-I$ demixing is excluded. In 3D rod-rod mixtures, where $I-I$ demixing can be stable [60], $\Delta_{I}$ is two order of magnitude larger than the maximal value of $\Delta_{I}$ obtained for $2 \mathrm{D}$ needle-disk mixture. To our best knowledge, there are no experimental and simulation studies predicting $I-I$ demixing transition in $2 \mathrm{D}$ hard body mixtures.

Regarding the reliability of the SPT, our results for demixing and ordering behaviour of hard superellipses are consistent with the results obtained for both thermal and athermal 2D binary mixtures using simulation and experimental methods. The size and shape differences are shown to induce attractive (depletion) forces between the large components of the binary mixture. As a result the segregation is observed in several 2D athermal and thermal binary mixtures such as the square-square [57], disk-disk [58, 92], disk-square [59], disk-rod [93-95] and confluent biological tissues [96]. We are not aware of experimental and simulation studies of mesogenic rod-rod studies, but the weakly anisotropic rod-rod mixtures serve also interesting transitions and orientational ordering features both in the athermal and thermal cases with glass transitions and jamming [78, 97, 98]. Our results are consistent with the results obtained for some monodisperse systems, where the fluid-solid transitions of hard disks and the $I-N$ transitions of hard ellipses become first-order with decreasing temperature if attractive interactions are also present between the particles $[99,100]$. This change can also be seen in the phase diagram of the mixture of superellipses where the second-order $I-N$ transition changes to be first-order due to the unlike excluded volume interactions, which corresponds to an attractive depletion interaction between the large superellipses.

The stability of tetratic ordering has been examined in the mixture of squares and superellipses. With adding hard squares into the sea of ordered superellipses we have found that the superellipses act as a quadrupolar ordering field for the squares. The observed phase is nematic for superellipses, while it is tetratic for squares. The strength of the ordering field is very weak, so the tetratic order parameter of the squares is low. To induce stronger tetratic order, the pressure should be increased substantially. The interesting feature of the tetratic order in binary mixtures is that the minimal packing fraction can be much lower than in the pure systems of squares [23] and other shapes [101,102]. Therefore, mixing of long particles with 
squares open the window for the detection of low density tetratic ordering in simulations and experiments.

In the presented phase diagrams the deformation parameters are taken to be even numbers, although the calculations can be performed for any real numbers of $n_{1} \geq 2$ and $n_{2} \geq 2$. This is due to the general form of the excluded area presented in the Appendix. This allows to get a deeper insight into the effect of varying deformation parameter. We note that our method allows to determine the distance of closest approach (contact distance) between two arbitrary superellipses. This distance is crucial in testing the overlap between the particles, which is used Monte Carlo simulations [16, 40] in perturbation theories of liquid crystals [103]. We believe that our expressions for the excluded area and the distance of closest approach between two superellipses will aid the future theoretical and simulation studies to understand the shape and size dependence of 2D melting, orientational ordering and demixing transitions.

\section{References:}

[1] K. J. Lee, J. Yoon, and J. Lahann, Current Opinion in Colloid \&Interface Science 16, 195 (2011).

[2] S. Sacanna, D. J. Pine, and G.R. Yi, Soft Matter 9, 8096 (2013).

[3] K. Zhao and T. G. Mason, Reports on Progress in Physics 81,126601 (2018).

[4] M. P. Allen, G. T. Evans, D. Frenkel, and B. M. Mulder, Advances in Chemical Physics 86, 1 (1993).

[5] S. C. McGrother, D. C. Williamson, and G. Jackson, J. Chem. Phys. 104, 6755 (1996).

[6] P. Bolhuis and D. Frenkel, J. Chem. Phys. 106, 666 (1997).

[7] P. J. Camp, C. P. Mason, M. P. Allen, A. A. Khare, and D. A. Kofke, J. Chem. Phys. 105, 2837 (1996).

[8] Y. Martínez-Ratón and E. Velasco, J. Chem. Phys. 129, 054907 (2008).

[9] G. W. Delaney and P. W. Cleary, Europhys. Lett. 89, 34002 (2010).

[10] F. J. Vesely, J. Chem. Phys. 141, 064109 (2014).

[11] J. M. Meijer, A. Pal, S. Ouhajji, H. N. Lekkerkerker, A. P. Philipse, and A. V. Petukhov, Nature Communications 8, 14352 (2017).

[12] K. Zhao, R. Bruinsma, and T.G. Mason, Proceedings of the National Academy of Sciences 108, 2684 (2011).

[13] K.W. Wojciechowski and D. Frenkel, Comp. Methods in Science and Tech. 10, 235 (2004). 
[14] A. Donev, J. Burton, F. H. Stillinger, and S. Torquato, Phys. Rev. B 73, 054109 (2006).

[15] L. Walsh, and N. Menon, J. Stat. Mech. Theory and Experiment 8, 083302 (2016).

[16] C. Avendano and F. A. Escobedo, Soft Matter 8, 4675 (2012).

[17] V. Narayan, N. Menon, and S. Ramaswamy, J. Stat. Mech.: Theory Exp. P01005 (2006).

[18] Y. Martínez-Ratón, E. Velasco, and L. Mederos, J. Chem. Phys. 125, 014501 (2006).

[19] K. Zhao, R. Bruinsma, and T. G. Mason, Nature Communications 3, 801 (2012).

[20] A.P. Gantapara, W. Qi, and M. Dijkstra, Soft Matter 11, 8684 (2015).

[21] M. R. Khadilkar and F. A. Escobedo, Soft Matter 12, 1506 (2016).

[22] Y. Martínez-Ratón, A. Díaz-De Armas, and E. Velasco, Phys. Rev. E 97, 052703 (2018).

[23] J. A. Anderson, J. Antonaglia, J. A. Millan, M. Engel, and S. C. Glotzer. Phys. Rev. X 7, 021001 (2017).

[24] J. M. Kosterlitz and D. J. Thouless, J. Phys. C 6, 1181 (1973); D. R. Nelson and B. I. Halperin, Phys. Rev. B 19, 2457 (1979).

[25] E. P. Bernard and W. Krauth, Phys. Rev. Lett. 107, 155704 (2011).

[26] D. Florea and H. M. Wyss, J. Colloid Interface Sci. 416, 30 (2014).

[27] Z. Zheng, F. Wang, and Y. Han, Phys. Rev. Lett. 107, 065702 (2011).

[28] C. K. Mishra, A. Rangarajan, and R. Ganapathy, Phys. Rev. Lett. 110, 188301 (2013).

[29] R. Duggal, M. Pasquali, Phys. Rev. Lett. 96, 246104 (2006).

[30] A. Czogalla, E. P. Petrov, D. J. Kauert, V. Uzunova, Y. Zhang, R. Seidel, and P. Schwille, Faraday Discuss. 161, 31 (2013).

[31] A. Czogalla, D. J. Kauert, R. Seidel, P. Schwille, and E. P. Petrov, Nano Lett. 15, 649 (2015).

[32] J. A. Cuesta and D. Frenkel, Phys. Rev. A 42, 2126 (1990).

[33] M. A. Bates and D. Frenkel, J. Chem. Phys. 112, 10034 (2000).

[34] D. Frenkel and R. Eppenga, Phys. Rev. A 31, 1776 (1985).

[35] R. Vink, Phys. Rev. Lett. 98, 217801 (2007).

[36] H. H. Wensink and R. Vink, Journal of Physics: Condensed Matter 19, 466109 (2007).

[37] R. Vink, Eur. Phys. J. B 72, 225 (2009). 
[38] M. Dijkstra and D. Frenkel, Phys. Rev. E 50, 349 (1994).

[39] A. Milchev, S. A. Egorov, and K. Binder, Soft Matter 13, 1888 (2017); A. Milchev and K. Binder, Nano Lett. 17, 4924 (2017).

[40] E. Bautista and G. Odriozola, J. Chem. Phys. 140, 204502 (2014).

[41] E. Basurto, P. Gurin, S. Varga, and G. Odriozola, Phys. Rev. Res. 2, 013356 (2020).

[42] T. Müller, D. de las Heras, I. Rehberg, and K. Huang, Phys. Rev. E 91, 062207 (2015).

[43] Y. Jiao, F.H. Stillinger, and S. Torquato, Phys. Rev. Lett. 100, 245504 (2008).

[44] O. Gromenko and V. Privman, Phys. Rev. E 79, 042103 (2009).

[45] B. N. Aleksić, N. M. Švrakić, and M. Belić, Phys. Rev. E 88, 062112 (2013).

[46]N. M. Švrakić, B. N. Aleksić, and M. R. Belić, Physica A 441, 93 (2016).

[47] S. M. Arifuzzaman, K. Dong, Q. Hou, H. Zhu, and Q. Zeng, Powder Technology 361, 112 (2020).

[48] J. Lin, H. Chen, L. Liu, and R. Zhang, Physica A 544, 123564 (2019).

[49] X. Zheng and P. Palffy-Muhoray, Phys. Rev. E 75, 061709 (2007).

[50] H. Schlacken, H. J. Mogel, and P. Schiller, Molecular Physics. 93, 777 (1998).

[51] T. Geigenfeind and D. de las Heras, J. Chem. Phys. 150, 184906 (2019).

[52] J. M. Taylor, Physics A 52, 095002 (2019).

[53] J. A. Millan, D. Ortiz, G. Van Anders, and S. C. Glotzer, ACS Nano 8, 2918 (2014).

[54] J. Russo and Nigel B. Wilding, Phys. Rev. Lett. 119, 115702 (2017).

[55] A. Santos, S. B. Yuste, M. López de Haro, and V. Ogarko, Phys. Rev. E 96, 062603 (2017).

[56] A. Díaz-De Armas and Y. Martínez-Ratón, Phys. Rev. E 95, 052702 (2017).

[57] A. Buhot and W. Krauth, Phys. Rev. E. 59, 2939 (1999).

[58] J. T. Kindt, J. Chem. Phys. 143, 124109 (2015).

[59] B. P. Prajwal and F. A. Escobedo, https://arxiv.org/abs/2004.02732.

[60] M. Dijkstra, Phys. Rev. E 58, 7523 (1998).

[61] R. Tenne and E. Bergmann, J. Chem. Phys. 70, 1952 (1978).

[62] R. J. Bearman and R. M. Mazo, J. Chem. Phys. 88, 1235 (1988).

[63] J. Talbot, J. Chem. Phys. 106, 4696 (1997). 
[64] F. Saija and P. V. Giaquinta, J. Chem. Phys. 117, 5780 (2002).

[65] F. Saija, Physical Chemistry Chemical Physics. 13, 11885 (2011).

[66] G. Fiumara, O. D. Pandaram, G. Pellicane, and F. Saija, J. Chem. Phys. 141, 214508 (2014).

[67] G. Fiumara, F. Saija, G. Pellicane, M. López de Haro, A. Santos, and S.B. Yuste, J. Chem. Phys. 147, 164502 (2017).

[68] W. T. Góźdź and A. Ciach, Condensed Matter Physics 19, 13002 (2016).

[69] E. Z. Hamad and G. O. Yahaya, Fluid Phase Equilibria.168, 59 (2000).

[70] R. van Roij and B. Mulder, Phys. Rev. E 54, 6430 (1996).

[71] M. Dijkstra and R. van Roij, Phys. Rev. E 56, 5594 (1997).

[72] K. R. Purdy, S. Varga, A. Galindo, G. Jackson, and S. Fraden, Phys. Rev. Lett. 94, 057801 (2005).

[73] Y. Martínez-Ratón, E. Velasco, and L. Mederos, Phys. Rev. E 72, 031703 (2005).

[74] D. de las Heras, Y. Martínez-Ratón, and E. Velasco, Phys. Rev. E 76, 031704 (2007).

[75] Y. Martinez-Raton, Liquid Crystals. 38, 697 (2011).

[76] L. Mederos, E. Velasco, and Y. Martínez-Ratón, J. Phys.: Condens. Matter 26, 463101 (2014).

[77] Wen-Sheng Xu, Zhao-Yan Sun and Li-Jia An, J. Chem. Phys. 142, 224506 (2015).

[78] Wen-Sheng Xu, Zhao-Yan Sun and Li-Jia An, Soft Matter. 11, 627 (2015).

[79] H. Reiss, H. L. Frisch, and J. L. Lebowitz, J. Chem. Phys. 31, 369 (1959).

[80] J. L. Lebowitz, E. Helfand, and E. Praestgaard, J. Chem. Phys. 43, 774 (1965).

[81] R. Kayser and H. Ravaché, Phys. Rev. A 17, 2067 (1978).

[82] B. Mulder, Phys. Rev. A 39, 360, (1989).

[83] P. van der Schoot, J. Chem. Phys. 106, 2355 (1997).

[84] H. N. W. Lekkerkerker, Ph. Coulon, R. van der Haegen, and R. Deblieck, J. Chem. Phys. 80, 3427 (1984).

[85] G. J. Vroege and H. N. W. Lekkerkerker, J. Phys. Chem. 97, 3601 (1993).

[86] K. Shundyak and R. van Roij, Phys. Rev. E 68, 061703 (2003).

[87] J. Kundu, J. Stilck, and R. Rajesh, Europhys. Lett. 112, 66002 (2015).

[88] R. van Roij, B. M. Mulder, and M. Dijkstra, Physica A 261, 347 (1998).

[89] H. H. Wensink and G. J. Vroege, Journal of Physics: Condensed Matter 16, S2015 (2004) 
[90] S. Varga, K. Purdy, A. Galindo, S. Fraden and G. Jackson Phys. Rev. E 72, 051704 (2006).

[91] T. Drwenski, P. Hooijera and R. van Roij, Soft Matter 12, 5684 (2016).

[92] P. Melby, A. Prevost, D. A. Egolf, and J. S. Urbach, Phys. Rev. E 76, 051307 (2007).

[93] A. Baldassarri, A. Puglisi, and A. Sarracino, C. R. Physique 16, 291 (2015).

[94] G. M. Rodríguez-Liñán, Y. Nahmad-Molinari, and G. Pérez-Ángel, PLoS ONE 11, e0156153 (2016).

[95] T. A. Marschall and S. Teitel, https://arxiv.org/pdf/2002.11191.pdf.

[96] P. Sahu, D. M. Sussman, M. Rübsam, A. F. Mertz, V. Horsley, E. R. Dufresne, C. M. Niessen, M. C. Marchetti, M. L. Manning, and J. M. Schwarz, Soft Matter, 16, 3325 (2020).

[97] L. Wang, B. Mei, J. Song, Y. Lu, and L. An, Science China Chemistry 61, 613 (2018).

[98] T. Marschall, Y.-E. Keta, P. Olsson, and S. Teitel, Phys. Rev. Lett. 122, 188002 (2019).

[99] Yan-Wei Li and M. P. Ciamarra, Phys. Rev. Lett. 124, 218002 (2020).

[100] P. Kundu, P. Mishra, A. Jaiswal, and J. Ram, J. Mol. Liquids 296, 111769 (2019).

[101] T. Terao, J. Chem. Phys 139, 134501 (2013).

[102] Z. Hou, Y. Zong, Z. Sun, F. Ye, T. G. Mason, and K. Zhao, Nature Communications 11, 2064 (2020).

[103] S. Varga, I. Szalai, J. Liszi, and G. Jackson, J. Chem. Phys. 116, 9107 (2002).

\section{ACKNOWLEDGMENTS}

H.S. and S.M. are grateful to the Research Council of Shahid Chamran University of Ahvaz for financial support (Grant No. SP98.490) and R.A. thanks Fasa University for supporting the research and providing computing facilities. S.M. also appreciates Institute of Physics and Mechatronics, University of Pannonia, Hungary. S.V. and P. G. acknowledge the financial supports of the National Research, Development, and Innovation Office (Grant No. NKFIH K124353) and Mexican CONACyT (Grant No. A1-S-9197). 


\section{Appendix: Excluded area between two superellipses}

The equation of a standard superellipse, where the center of the body is fixed at the origin and the semi-major axis $a$ (the semi-minor axis $b$ ) is parallel with axis $x(y)$, can be written as

$\Phi(x, y)=1$

where

$\Phi(x, y)=\left(\frac{|x|}{a}\right)^{n}+\left(\frac{|y|}{b}\right)^{n}$.

Note that $n$ is the deformation parameter, which is a positive real number. A parametric representation of the above superellipse is given by

$\vec{r}(\theta)=r(\theta)\left(\begin{array}{c}\cos \theta \\ \sin \theta\end{array}\right)$,

where

$r(\theta)=\left[\left(\frac{|\cos \theta|}{a}\right)^{n}+\left(\frac{|\sin \theta|}{b}\right)^{n}\right]^{\frac{-1}{n}}$.

In Eqs.(A.3) and (A.4) $r$ is the usual radial distance and $\theta$ is the polar angle. Note that the curve of the superellipse is smooth only and the gradient is well defined at all points of the superellipse for $n \geq 2$ even if the absolute values of $x$ and $y$ are used in (A.2). Therefore we deal with $n \geq 2$ case. The normal unit vector of the curve at any point $\vec{r}$ is given by $\vec{n}(\vec{r})=\nabla \Phi(\vec{r}) /|\nabla \Phi(\vec{r})|$, which points out from the superellipse. Using our parametric representation, $\nabla \Phi(\vec{r})$ can be expressed as

$\nabla \Phi(\vec{r})=n[r(\theta)]^{n-1}\left(\begin{array}{l}\frac{|\cos \theta|^{n-2}}{a^{n}} \cos \theta \\ \frac{|\sin \theta|^{n-2}}{b^{n}} \sin \theta\end{array}\right)$

Let us continue with two superellipses, which are characterized by the parameters $a_{1}, b_{1}, n_{1}$ and $a_{2}, b_{2}, n_{2}$ and $\varphi_{12}$ is the angle between their main axes. We use the notations of Fig. 10, where $\vec{r}_{1}$ and $\vec{r}_{2}$ position vectors points to the common point of the two bodies, when they are 
in contact. The polar angles of these vectors are denoted by $\theta_{1}$ and $\theta_{2}$, which are measured from $x$ and $x^{\prime}$ axes of the respective body fixed coordinate systems. The angle between the two coordinate systems $\left(\varphi_{12}\right)$ is the angle between the two superellipses. In the following derivations, we determine the excluded areas for smooth superellipes only, i.e. $n_{1}$ and $n_{2} \geq 2$. Fig. 10 shows that the two curves have common tangent at the contact point. The normal vectors of both superellipses point outside from the corresponding curves and must have exactly the opposite directions, i.e. $\vec{n}_{1}\left(\vec{r}_{1}\right)=-\vec{n}_{2}\left(\vec{r}_{2}\right)$, because the particles touch each other from outside. As the particle 2 is rotated by $\varphi_{12}$ angle with respect to the particle 1 , its normal vector is also rotated by $\varphi_{12}$. Therefore the condition for the normal vectors becomes

$$
\frac{\vec{\nabla} \Phi_{1}\left(\vec{r}_{1}\left(\theta_{1}\right)\right)}{\left|\vec{\nabla} \Phi_{1}\left(\vec{r}_{1}\left(\theta_{1}\right)\right)\right|}=-\frac{\hat{R}\left(\varphi_{12}\right) \nabla \Phi_{2}\left(\vec{r}_{2}\left(\theta_{2}\right)\right)}{\left|\vec{\nabla} \Phi_{2}\left(\vec{r}_{2}\left(\theta_{2}\right)\right)\right|}
$$

where the rotational matrix $\hat{R}\left(\varphi_{12}\right)$ is given by

$$
\hat{R}\left(\varphi_{12}\right)=\left(\begin{array}{cc}
\cos \varphi_{12} & -\sin \varphi_{12} \\
\sin \varphi_{12} & \cos \varphi_{12}
\end{array}\right) .
$$

Note that the gradients are taken in the particle's fixed frames and that the rotation does not affect the norm of the vector. Substituting Eqs. (A.4) and (A.5) with the corresponding indices of the particles into Eq. (6) and using the rotational matrix Eq. (A.7), we get the following two equations for the $x$ and $y$ components of Eq. (A.6)

$$
\frac{\cos \theta_{1}\left|\cos \theta_{1}\right|^{n_{1}-2}}{\left[\left|\cos \theta_{1}\right|^{2\left(n_{1}-1\right)}+\left(\frac{a_{1}}{b_{1}}\right)^{2 n_{1}}\left|\sin \theta_{1}\right|^{2\left(n_{1}-1\right)}\right]^{\frac{1}{2}}}=\frac{-\cos \varphi_{12} \cos \theta_{2}\left|\cos \theta_{2}\right|^{n_{2}-2}+\left(\frac{a_{2}}{b_{2}}\right)^{n_{2}} \sin \varphi_{12} \sin \theta_{2}\left|\sin \theta_{2}\right|^{n_{2}-2}}{\left[\left|\cos \theta_{2}\right|^{2\left(n_{2}-1\right)}+\left(\frac{a_{2}}{b_{2}}\right)^{2 n_{2}}\left|\sin \theta_{2}\right|^{2\left(n_{2}-1\right)}\right]^{\frac{1}{2}}},
$$

and 
$\frac{\sin \theta_{1}\left|\sin \theta_{1}\right|^{n_{1}-2}}{\left[\left(\frac{b_{1}}{a_{1}}\right)^{2 n_{1}}\left|\cos \theta_{1}\right|^{2\left(n_{1}-1\right)}+\left|\sin \theta_{1}\right|^{2\left(n_{1}-1\right)}\right]^{\frac{1}{2}}}=\frac{-\sin \varphi_{12} \cos \theta_{2}\left|\cos \theta_{2}\right|^{n_{2}-2}-\left(\frac{a_{2}}{b_{2}}\right)^{n_{2}} \cos \varphi_{12} \sin \theta_{2}\left|\sin \theta_{2}\right|^{n_{2}-2}}{\left[\left|\cos \theta_{2}\right|^{2\left(n_{2}-1\right)}+\left(\frac{a_{2}}{b_{2}}\right)^{2 n_{2}}\left|\sin \theta_{2}\right|^{2\left(n_{2}-1\right)}\right]^{\frac{1}{2}}}$.

Eqs. (A.8) and (A.9) provide a relationship between $\theta_{1}$ and $\theta_{2}$ when the two particles are in contact. This means practically that, for a given relative orientation $\varphi_{12}$, supposing that the second particle touch the first one at $\vec{r}_{2}\left(\theta_{2}\right)$ point, the simultaneous solution of Eqs. (A.8) and (A.9) provides $\theta_{1}$ as a function $\theta_{2}$ and $\vec{r}_{1}\left(\theta_{1}\right)$ points to the contact point of the particle 1.The contact distance vector $\vec{\sigma}$, which connects the centers of the two particles when they are in contact, depends only on $\theta_{2}$, because $\vec{\sigma}=\vec{r}_{1}-\vec{r}_{2}$. Therefore, the excluded area between the first and second particles can be expressed from the well-known formula for the area of twodimensional objects as

$$
A_{e x c}=\frac{1}{2} \int_{0}^{2 \pi} \hat{e}_{z}\left(\vec{\sigma} \times \frac{d \vec{\sigma}}{d \theta_{2}}\right) d \theta_{2}
$$

where $\hat{e}_{z}$ is the unit vector perpendicular to the $x y$ plane, which selects the $z$ component of the cross product. Using $\vec{\sigma}=\vec{r}_{1}-\vec{r}_{2}$ and the identity of $-\vec{r}_{1} \times \frac{d \vec{r}_{2}}{d \theta_{2}}-\vec{r}_{2} \times \frac{d \vec{r}_{1}}{d \theta_{2}}=\frac{d\left(\vec{r}_{1} \times \vec{r}_{2}\right)}{d \theta_{2}}+2 \frac{d \vec{r}_{2}}{d \theta_{2}} \times \vec{r}_{1}$ we can rewrite Eq (A. 10) as

$$
A_{e x c}=\tilde{a}_{1}+\tilde{a}_{2}+\int_{0}^{2 \pi} \hat{e}_{z}\left(\frac{d \vec{r}_{2}}{d \theta_{2}} \times \vec{r}_{1}\right) d \theta_{2}
$$

where

$$
\begin{aligned}
\tilde{a}_{i} & =\frac{1}{2} \int_{0}^{2 \pi} \hat{e}_{z}\left(\vec{r}_{i} \times \frac{d \vec{r}_{i}}{d \theta_{2}}\right) d \theta_{2} \\
& =\frac{a_{i} b_{i}}{n_{i}} 2^{2\left(1-\frac{1}{n_{i}}\right)} \sqrt{\pi} \frac{\Gamma\left(\frac{1}{n_{i}}\right)}{\Gamma\left(\frac{1}{n_{i}}+\frac{1}{2}\right)}
\end{aligned}
$$


is the area of particle $i$. Note that in the derivation of Eq. (A.11) we have performed an integration using the integration by parts method. To compute the last term of Eq. (A.11) we need the components of $\vec{r}_{1}$ and $\vec{r}_{2}$ relative to the same coordinate frame. Using the coordinate frame, which is fixed to the particle 1, the derivate in Eq. (A11) is given by

$$
\frac{d \vec{r}_{2}}{d \theta_{2}}=\hat{R}\left(\varphi_{12}\right)\left[\frac{d r_{2}}{d \theta_{2}}\left(\begin{array}{c}
\cos \theta_{2} \\
\sin \theta_{2}
\end{array}\right)+r_{2}\left(\begin{array}{c}
-\sin \theta_{2} \\
\cos \theta_{2}
\end{array}\right)\right] .
$$

Using this equation, the final form of the excluded area can be written as

$$
\begin{aligned}
A_{\text {exc }}= & \tilde{a}_{1}+\tilde{a}_{2}+\int_{0}^{2 \pi} d \theta_{2}\left(\left[\frac{d r_{2}}{d \theta_{2}} \cos \left(\theta_{2}+\varphi_{12}\right)-r_{2} \sin \left(\theta_{2}+\varphi_{12}\right)\right] \sin \left(\theta_{1}\right)\right. \\
& \left.-\left[\frac{d r_{2}}{d \theta_{2}} \sin \left(\theta_{2}+\varphi_{12}\right)+r_{2} \cos \left(\theta_{2}+\varphi_{12}\right)\right] \cos \left(\theta_{1}\right)\right) r_{1},
\end{aligned}
$$

Here we used the central symmetry of the excluded area, therefore the integral over $\theta_{2}$ runs only up to $\pi$. In the above expression $\theta_{1}, r_{1}$ and $r_{2}$ are considered to be functions of $\theta_{2}$ from the solution of Eqs. (A.8)-(A.9). Note that we need only $\sin \theta_{1}$ and $\cos \theta_{1}$ as functions of $\theta_{2}$ in the excluded area calculations (A.14). Now we show that it is possible to get analytic formulas for $\sin \theta_{1}$ and $\cos \theta_{1}$ in the following way. Let us denote the r.h.s of Eqs. (A.8) and (A.9) by $F$ and $G$, respectively, which functions depend on the molecular parameters of particle 2, $\theta_{2}$ and $\varphi_{12}$. Using $F$ we get from Eq. (A. 8) that

$$
\left(\cos ^{2} \theta_{1}\right)^{n_{1}-1}=\left[\left(\cos ^{2} \theta_{1}\right)^{n_{1}-1}+\left(\frac{a_{1}}{b_{1}}\right)^{2 n_{1}}\left(1-\cos ^{2} \theta_{1}\right)^{n_{1}-1}\right] F^{2}
$$

which can be written shortly as

$$
\cos ^{2} \theta_{1}=\frac{c}{1+c}
$$

where

$$
c=\left[\frac{F^{2}}{1-F^{2}}\left(\frac{a_{1}}{b_{1}}\right)^{2 n_{1}}\right]^{\frac{1}{n_{1}-1}}
$$


From Eq. (A.8) it is clear that the sign of $\cos \theta_{1}$ is the same as the sign of $F$, therefore

$$
\cos \theta_{1}=\operatorname{sgn}(F) \sqrt{\frac{c}{1+c}}
$$

In a similar way from Eq. (A.9) we get that

$$
\sin \theta_{1}=\operatorname{sgn}(G) \sqrt{\frac{b}{1+b}}
$$

where

$$
b=\left[\frac{G^{2}}{1-G^{2}}\left(\frac{b_{1}}{a_{1}}\right)^{2 n_{1}}\right]^{\frac{1}{n_{1}-1}} .
$$

We must mention here that Eqs. (A.18) and (A.19) are consistent with each other satisfying the identity $\cos ^{2} \theta_{1}+\sin ^{2} \theta_{1}=1$. Substituting Eqs. (A.18) and (A.19) into Eq. (A.14) the excluded area is expressed explicitly as a function of $\theta_{2}$. Using these tricks the integrals can be performed easily using a simple numerical quadrature without resorting to equation solvers for Eqs. (A.8) and (A.9). By the appropriate choice of the molecular parameters Eq. (A14) can provide the like $\left(A_{e x c}^{11}, A_{e x c}^{22}\right)$ and the unlike $\left(A_{e x c}^{12}, A_{e x c}^{21}\right)$ excluded areas. If particle 1 and particle 2 are identical, i.e. both particles are characterized with $\left(a_{1}, b_{1}, n_{1}\right)$ or $\left(a_{2}, b_{2}, n_{2}\right)$ we get $A_{e x c}^{11}$ or $A_{e x c}^{22}$ .However we get $A_{e x c}^{21}$ and $A_{e x c}^{12}$ if the particles have different parameters, i.e. $\left(a_{1}, b_{1}, n_{1}\right) \neq\left(a_{2}, b_{2}, n_{2}\right)$. 


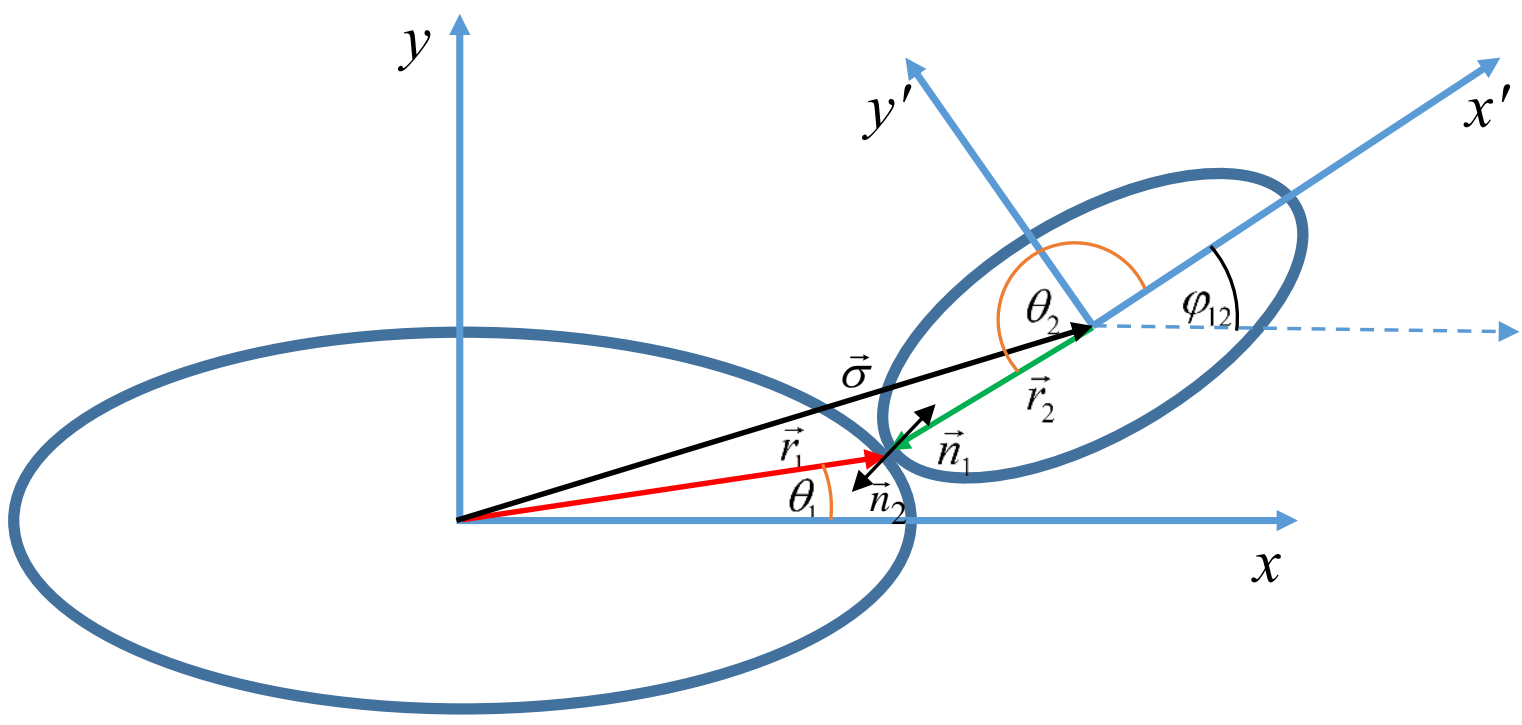

Figure 10: Two superellipses are in contact externally. The following notation is used: the angle between the major axes of the two superellipses is denoted by $\varphi_{12}, \vec{\sigma}$ is the contact distance vector between the two superellipses, $\theta_{1}$ and $\theta_{2}$ are the orientations of the position vectors $\vec{r}_{1}$ and $\vec{r}_{2}$ of the contact point which are measured in the coordinate frame fixed to particle 1 and 2, respectively. Moreover $\vec{n}_{1}$ and $\vec{n}_{2}$ are the normal vectors of the common tangent.

\section{Data Availability Statement}

The data that support the findings of this study are available from the corresponding author upon reasonable request. 